\title{
Mathematical modeling of the process of sustainable development of the personnel potential of industrial universities
}

\author{
Natalia Kalganova* \\ Ural State University of Railway Transport, 66 Kolmogorova str., Yekaterinburg, Russia, 620034
}

\begin{abstract}
The paper considers mathematical differential models of the dynamics of the number of teaching staff of a railway university during a certain period of its functioning. With the help of the constructed systems of ordinary differential equations, a method for managing the sustainable development of the personnel potential of industrial universities is proposed. In his research, the author relied on the works of Russian scientists, in particular, on [1-5]. To implement the principle of the scientific approach, the article proposes a method for studying the regularities of the dynamics of the number of university faculty members using mathematical tools and mathematical modeling. Modeling in this case allows us to identify some patterns and contribute to the management of the sustainable development of the personnel potential of railway universities. The paper presents an analytical solution of the Cauchy problem for a system of differential equations with given initial conditions. The model built on the basis of differential equations has practical value, because it allows you to determine and predict the number of personnel of the university at a specified time. In this paper, based on the methods of statistical analysis, the composition of PPS by age is studied. Some negative trends in the groups of teachers that create risks in the provision of the university with highly qualified personnel are identified.
\end{abstract}

\section{Introduction}

When solving the problems of technological, socio-economic and cultural development of the country, industries and regions, it is necessary to rely on science - universities and research institutes. Such support guarantees sustainable economic growth. The development of any industry (in our case, the railway) becomes sustainable if the branch university is formed on the basis of three blocks: the material and technical base, stable sustainable financing and the availability of highly qualified personnel.

To ensure the dynamic development of these blocks, it is necessary to implement program-targeted management of planning and forecasting at the university on a scientific basis. To create a system of regulation of all processes in the university, it is necessary to develop and implement a targeted program of personnel policy, including methods for managing the strategic development of the university's personnel potential for a certain

\footnotetext{
${ }^{*}$ Corresponding author: kalganova.natalia@yandex.ru
} 
period. The main thing in such a program is a system of planning and forecasting, a monitoring system that records the implementation of target parameters, the functions of the program are assigned to the department, management and specific persons from the university administration.

The main purpose of this work is to solve the problem of constructing a mathematical model of the dynamics of the number of teaching staff for a certain period of the university's functioning.

The article presents a mathematical model in the form of a system of linear inhomogeneous differential equations with constant first-order coefficients, on the basis of which it is required to find an analytical and numerical solution of the system that simulates the dynamics of the PPS., to determine its parameters corresponding to the rational survey mode.

To solve this problem, we use analytical methods of the theory of systems of first-order ordinary differential equations and methods for solving them in MathCAD 15 [5-6].

To build a differential mathematical model, we study the initial information, the analysis of statistical data, and the patterns of interaction. The derivation of differential equations is based on taking into account real regularities.

\section{Building a mathematical model}

Denote $\mathrm{N}(\mathrm{t})$-the number of scientific and pedagogical staff of the university according to the staffing table at the given time $\mathrm{t}$. Here it is necessary to determine exactly-what number $\mathrm{N}$ are we considering? At the university, the rates are determined in accordance with the norms. The number of teaching staff providing the educational process can be greater than the number of rates, i.e. $\mathrm{N}>\mathrm{S}$, since both internal and external part-time employees work at the university.

In addition, part of the PPP may work part-time. Although the proposed mathematical model can describe any of these options, we will investigate the dynamics of teaching staff, noting that the number $N(t)$ - is a stable teaching team formed over the past few years, consisting of full - time, internal and external teachers. In accordance with the structure of the teaching staff, we divide $N(t)$ into three subgroups: $x(t)$ - the number of teachers in the positions of assistants, teachers, and senior teachers; $y(t)$ - he second group, which includes teachers with a $\mathrm{PhD}$ degree, the title of associate professor; $z(t)$ - which includes doctors of science, professors. Each teacher, including external part-time teachers, is a member of one of the groups. The quantitative composition of each group can change at any time, but the peak of changes occurs mainly after the end of the school year. Therefore, we will consider the functions $x(t), y(t), z(t)$ as continuous, differentiable, and positive. The total number of PPS N (t) is represented by the sum:

$$
N(t)=x(t)+y(t)+z(t)
$$

Consider the first group of teaching staff, the number of which is $x(t)$ Consider the first group of teaching staff, the number of which is $x(t)$ people and changes over time $t$. The rate of change is proportional to the number of teachers $-\alpha_{1} x(t)$, which is associated with the transition of part of the teaching staff to the second group, equal to $-\beta_{1} y(t)$, which is associated with the transition of part of the teaching staff to the second group, equal to- $\beta 1 \mathrm{y}(\mathrm{t})$, retirement, dismissal, etc. The rate will change due to the arrival of newly accepted $f_{l}(t)$. teachers. Thus, for the first group of PPS, we obtain the differential equation:

$$
\frac{d x}{d t}=-\alpha_{1} x(t)-\beta_{1} y(t)+f_{1}(t)
$$


Consider the second group of PPS. The rate of change in this group depends on the speed reduction reduction $-\alpha_{2} y(t)$ due to layoffs and other reasons, then the velocity of the second group will decline because of the transition of teachers in the third group in proportion to the value $-\beta_{2} z(t)$, dismissal, etc., at the same time, the growth rate of this group will increase because of the transition of employees from the first group by the value $\gamma_{1} x(t)$ and the new $\operatorname{method} f_{2}(t)$.

Analytically, this analysis can be written as the equation

$$
\frac{d y(t)}{d t}=-\alpha_{2} y(t)-\beta_{2} z(t)+\gamma_{1} x(t)+f_{2}(t)
$$

The coefficients $\alpha_{1}, \alpha_{2}, \beta_{1}, \beta_{2}, \gamma_{1}, \gamma_{2}, \gamma_{3}$ of the system of differential equations are positive constants that regulate the retirement of the PPP due to the dismissal/increase of the group.

Similarly, we construct a third equation for the third group and obtain an inhomogeneous system of ordinary differential equations:

$$
\begin{gathered}
\frac{d x(t)}{d t}=-\alpha_{1} x(t)-\beta_{1} y(t)+f_{1}(t) \\
\frac{d y(t)}{d t}=-\alpha_{2} \gamma(t)-\beta_{2} z(t)+\gamma x(t)+f_{2}(t) \\
\frac{d z(t)}{d t}=-\alpha_{3} z(t)+\beta_{3} \gamma(t)+f_{3}(t)
\end{gathered}
$$

This process is discrete in nature and describes the dynamics of specific objects, but the experience of analyzing discrete objects allows us to describe processes using continuous functions $x(t), y(t), z(t), f_{i}(t)$, where $i=1,2,3$, which are continuous positive functions having continuous first-order derivatives. Coefficients $\alpha_{i}, \beta_{i}, y-$ positive constants, which are determined on the basis of statistical analysis of the number of PPS.

We introduce the matrix A of coefficients corresponding to the system of linear differential equations (4):

$$
\mathrm{A}=\left(\begin{array}{ccc}
-\alpha_{1} & -\beta_{1} & 0 \\
\gamma & -\alpha_{2} & -\beta_{2} \\
0 & \beta_{3} & -\alpha_{3}
\end{array}\right)
$$

Additionally, we introduce two matrices:

- matrix-column of unknown functions

$$
X=\left(\begin{array}{l}
x(t) \\
y(t) \\
z(t)
\end{array}\right)
$$

- matrix - column of summary members

$$
F=\left(\begin{array}{l}
f_{1}(t) \\
f_{2}(t) \\
f_{3}(t)
\end{array}\right)
$$

With the help of the entered matrices, system (4) can be briefly written in matrix form: 


$$
\frac{d}{d t} X=A X+F
$$

Consider the matrices $\mathrm{A}$ and $\mathrm{F}$, whose elements have a set of adjustable parameters that affect the optimal control of the solution of the system of ordinary differential equations (4) or (8). By varying the elements of the matrix (coefficients of the system) A and the function $f_{\mathrm{i}}(t)$ (free members of the system), it is possible to achieve an increase or decrease in the rate of change in the number of faculty members in each group and for a certain period of time to achieve an increase (decrease) in the number of university teachers.

Let's introduce a definition. We call matrices (5) - (7) and the elements of these matrices the control of the solution of the system of ordinary differential equations (4) or (8), i.e., this is a set of control parameters from the set of elements of matrix $A$ and time functions of the matrix $F$, which have a direct impact on the solution of the system (4), which simulates the management of long-term development and the achievement of target results of the human resources potential of the industry-specific university.

\section{Investigation of a mathematical model and solution of the Cauchy problem for a system of differential equations (4)}

For systems (4), (8), we formulate a problem with initial conditions for $t=0$ :

$$
x(0)=x_{0}, y(0)=y_{0}, z(0)=z_{0}
$$

where $N_{0}$ - the number of PPS for a certain initial period.

With, $x(0)+y(0)+z(0)=N_{0}$

A system of linear inhomogeneous equations with constant coefficients is integrated by reducing it to a single equation of a higher order.

So, expressing the unknown function from the first equation $y(t)$, after the introduction, which is in the second and third equations of system (4), we get two equations with unknowns $x(t)$ и $z(t)$. If there is no simple and obvious solution to two equations with two unknowns, then the elimination process can be continued. Finally, the resulting equation of a higher order, namely the third order, will be linear with constant coefficients. The solution of the resulting equation is also quite difficult, which is associated with the consideration of variants of the roots of the characteristic equation. In the future, we will use the MathCAD 15 package to analyze options for optimal management of human resource development using control operators [7]. The analysis shows that system (4) can be radically simplified without violating the generality of the solution. To do this, it is enough to assume that with a decrease in the number of faculty members of the university in each group, there are all possible reasons: retirement, dismissal in connection with the transfer of part of the faculty to the second and third groups after the defense of dissertations, etc. Then the system (4) will take a simplified form:

$$
\begin{gathered}
\frac{d x}{d t}=-\alpha_{1} x(t)+f_{1}(t) \\
\frac{d y(t)}{d t}=-\alpha_{2} y(t)+\beta_{1} x(t)+f_{2}(t) \\
\frac{d z(t)}{d t}=-\alpha_{3} z(t)+\beta_{2} y(t)+f_{3}(t)
\end{gathered}
$$


Let us prove the existence and uniqueness theorem for the solution of the Cauchy problem for system (10) of differential equations with initial conditions (9). Let on some closed segment $t \in[0, \mathrm{~T}]$, the functions $f_{\mathrm{i}}(t), i=1,2,3$ the functions $\mathrm{fi}(\mathrm{t}), \mathrm{i}=1,2,3$ are continuous, then the Cauchy problem for an inhomogeneous system of first-order differential equations (9)-(10) will have a unique bounded solution:

$$
x_{k}(t)=\ell^{-\alpha_{k} t}\left[x_{k 0}+\int_{0}^{t}\left(\beta_{\mathrm{k}}-1 \varphi_{k}-1(\tau)+f_{k}(\tau)\right) \ell^{\alpha_{\mathrm{k}} \tau} d t\right]
$$

where $x_{k}$ are denoted by $x=x_{1}, y=x_{2}, z=x_{3}$ at $k=1,2,3 ; \varphi_{k}$ - the solution of the previous, relative to the number $k$ для $k=1,2,3$, differential equation of the system (7) at $\varphi_{0}=0 ; x_{k} 0$ $=\left\{x_{0}, y_{0}, z_{0}\right\}-$ initial conditions $(6)$ at $k=1,2,3$.

Proof

The first differential equation of the system is a first-order linear equation that has a solution:

$$
x(t)=\ell^{-\alpha_{1} t}\left[x_{0}+\int_{0}^{t} f_{1}(\tau) \ell^{\alpha_{1} \tau} d t\right]=\varphi_{1}(t)
$$

where the function $\varphi_{1}(t)$ - is the solution of the first equation.

The second equation, taking into account (11), is as follows:

$$
\frac{y(t)}{d t}+\alpha_{2} y(t)=\beta_{1} \varphi_{1}(t)+f_{2}(t)
$$

The resulting differential equation is also a first-order linear equation, and its solution $\varphi_{2}(t)$ has the form:

$$
y(t)=\ell^{-\alpha_{2} t}\left[y_{0}+\int_{0}^{t}\left(\beta_{1} \varphi_{1}(\tau)+f_{2}(\tau)\right) \ell^{\alpha_{2} \tau} d \tau\right]=\varphi_{2}(t)
$$

Similarly, we find a solution to the third equation:

$$
z(t)=\ell^{-\alpha_{3} t}\left[z_{0}+\int_{0}^{t}\left(\beta_{2} \varphi_{2}(\tau)+f_{3}(\tau)\right) \ell^{\alpha_{3} \tau} d \tau\right]=\varphi_{3}(t)
$$

Suppose that the functions $f_{1}(t), i=1,2,3$ are bounded, $\left|f_{i}(t)\right|<B, i=1,2,3$

Then for everyone $t €[0, \mathrm{~T}]$ we obtain the inequalities:

$$
\left|x_{1}(t)\right| \leq \ell^{-\alpha_{1} t}\left[x_{0}+\int_{0}^{t} \mid f_{1}(\tau) \ell^{\alpha_{1} \tau} d \tau\right] \leq \ell^{-\alpha t}\left[x_{0}+\frac{B}{\alpha_{1}} \ell^{\alpha_{1} t}(t)\right]=\ell^{-\alpha_{1} t} x_{0}+\frac{B}{\alpha_{1}} t \leq x_{0}+\frac{B}{\alpha_{1}} t<D=\text { const } t \in[0 . \mathrm{T}]
$$

Finally: $\left|x_{1}(t)\right|<D$

Similar inequalities can be obtained for all subsequent equations of the system (10).

If we assume the existence of two solutions $x_{1}(t)$ и $x_{2}(t)$ of the first equation of the system (10) with initial conditions (9), then the function $x(t)=x_{2}(t)-x_{1}(t)$ satisfies a homogeneous equation, that is $f_{1}=0$ and we have a zero initial condition. Then the formula (11) for solving the first homogeneous equation of the system (10) is expressed by the equality $x(t)=0$ hence the equality $x_{1}(t)=x_{2}(t)$. Similarly, it is easy to show the uniqueness of the solutions of the problems for the second and third equations of the system (10). Thus, there is only one solution for problem (9) - (10).

This proof method defines an algorithm for the analytical solution of the Cauchy problem for a system of differential equations (10) with initial conditions (9). 
Based on the above, it is proved:

Let the functions $f_{i}(t), i=1,2,3$ are continuous on some closed segment $t \in[0, T]$, then the Cauchy problem for a non-homogeneous system of first-order differential equations (9) - (10) has a unique bounded solution:

$$
x_{k}(t)=\ell^{-\alpha_{k} t}\left[x_{k 0}+\int_{0}^{t}\left(\beta_{k-1} \varphi_{k-1}(\tau)+f_{k}(\tau)\right) \ell^{\alpha_{k} \tau} d \tau\right]
$$

where $x_{k}$, denotes $x=x_{1}, y=x_{2}, z=x_{3}$ by $k=1,2,3$;

$\varphi_{k}$ - the solution of the previous one, with respect to the number $k$ for $k=1,2,3$, the differential equation of system (7) for $\varphi_{0}=0 ; x_{k 0}=\left\{x_{0}, y_{0}, z_{0}\right\}$-initial conditions (6) for $k=$ $1,2,3$.

\section{Numerical solution of systems of ordinary differential equations}

Consider system (10) with matrices of equations with constant elements of matrices A and $F$ using MathCad 15 (Fig.1).

Example 1.

$$
\begin{array}{r}
A=\left(\begin{array}{ccc}
-0.05 & 0 & 0 \\
0.01 & -0.01 & 0 \\
0 & 0.003 & -0.04
\end{array}\right), F=\left(\begin{array}{c}
0.15 \\
0.043 \\
0.01
\end{array}\right) . \\
x(0)=3
\end{array}
$$

\section{Given}

$$
\begin{aligned}
& \frac{d}{d t} x(t)=-0.05 x(t)+0.1 s \\
& \frac{d}{d t} y(t)=-0.01 \cdot y(t)+0.01 \cdot x(t)+0.04 \\
& \frac{d}{d t} z(t)=-0.04 z(t)+0.003 y(t)+0.01
\end{aligned}
$$

$$
y(0)=6
$$

$$
z(0)=1
$$$$
\left(\begin{array}{l}
x \\
y \\
z
\end{array}\right):=\text { Odesolve }\left[\left(\begin{array}{l}
x \\
y \\
z
\end{array}\right), t, 10\right]
$$

Target values at $t=10: x(10)=3$, $y(10)=6,124, z(10)=0,903$ 


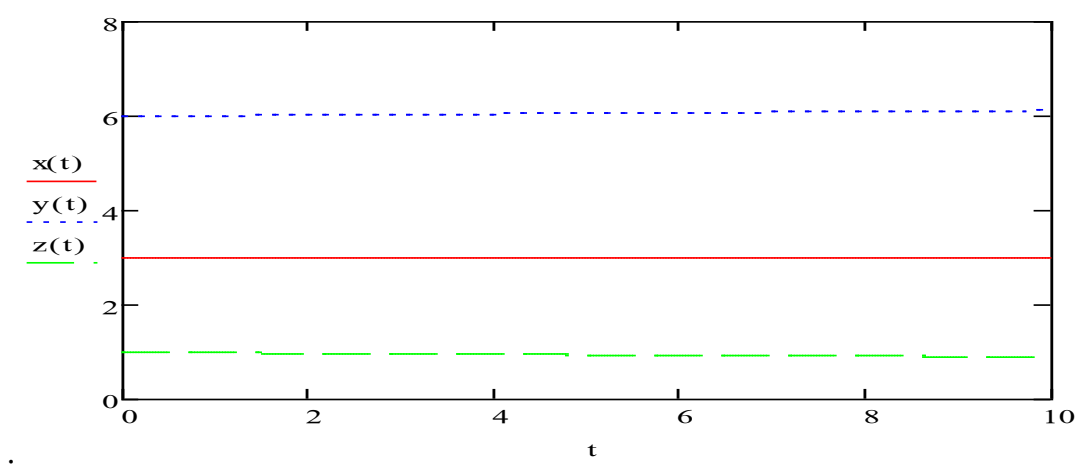

Fig. 1. System solution plots (10).

Figure 1 shows that if the unknown function $f(x)=$ const, then, in the initial period and in the subsequent period, the number of teachers stabilizes and becomes unchanged. 2.

Let us consider using MathCad 15 a system graph with control matrices (Fig. 2).Example

$$
\begin{gathered}
A=\left(\begin{array}{ccc}
-0.05 & 0 & 0 \\
0.025 & -0.021 & 0 \\
0 & 0.02 & -0.05
\end{array}\right), F=\left(\begin{array}{c}
0.15 \\
0.1 \\
0.01
\end{array}\right) . \\
\frac{\mathrm{d}}{\mathrm{dt}} \mathrm{z}(\mathrm{t})=-0.05 \mathrm{z}(\mathrm{t})+0.02 \cdot \mathrm{y}(\mathrm{t})+0.01
\end{gathered}
$$

Given

$$
\begin{array}{ll}
\frac{\mathrm{d}}{\mathrm{dt}} \mathrm{x}(\mathrm{t})=-0.05 \mathrm{x}(\mathrm{t})+0.1 \mathrm{~s} & \mathrm{x}(0)=2 \\
\frac{\mathrm{d}}{\mathrm{dt}} \mathrm{y}(\mathrm{t})=-0.021 \mathrm{y}(\mathrm{t})+0.025 \mathrm{x}(\mathrm{t})+0.1 & \mathrm{y}(0)=4 \\
& \mathrm{z}(0)=1
\end{array}
$$

$$
\left(\begin{array}{l}
x \\
y \\
z
\end{array}\right):=\text { Odesolve }\left[\left(\begin{array}{l}
x \\
y \\
z
\end{array}\right), t, 10\right]
$$




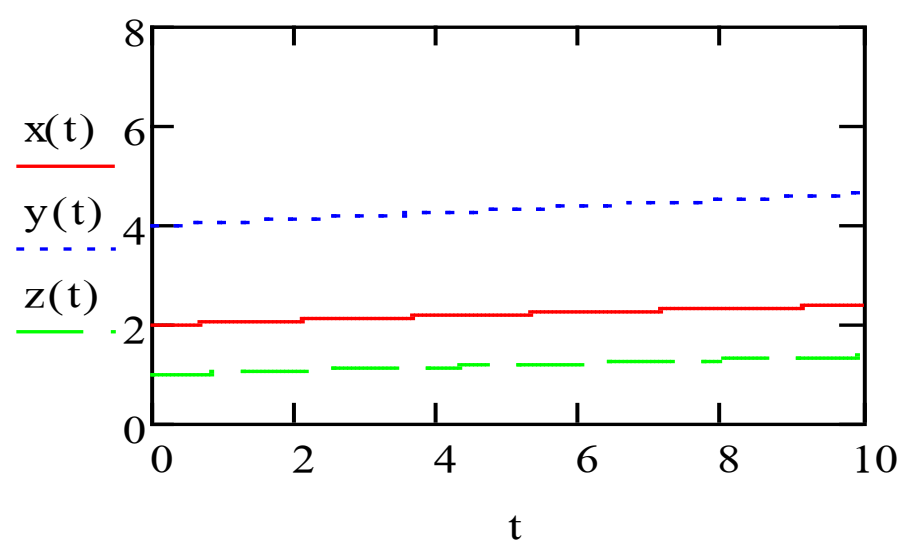

Fig. 2. System graph with control matrices

$$
x(10)=2,393 ; z(10)=1,37 ; y(10)=4,645
$$

Thus, examples 1 and 2 show that the control matrix (5) of the system of differential equations (4) is the most important mechanism in the development of management decisions on the regulation of teaching staff. Thus, changes in the elements of the management matrices of the system (5) have a direct impact on the decision of the system, which determines the process of managing the long-term development and achievement of target results of the personnel potential of the branch university.

For each group of teachers, there is a certain increase in the number of teaching staff.

Let us consider a scenario when there is a sharp decrease in the number of teaching staff at a university due to various reasons, including the sudden departure of part-time workers (Fig. 3).

Example 3 of a system with initial conditions $x(0)=1,8, y(0)=3,9, z(0)=0,9$, in which the control matrices for achieving the previous parameters are of the form:

$$
A=\left(\begin{array}{ccc}
-0.05 & 0 & 0 \\
0.025 & -0.021 & 0 \\
0 & 0.02 & -0.05
\end{array}\right), F=\left(\begin{array}{c}
0.1665 \\
0.12 \\
0.022
\end{array}\right)
$$

Given

$$
\frac{\mathrm{d}}{\mathrm{dt}} \mathrm{x}(\mathrm{t})=-0.05 \mathrm{x}(\mathrm{t})+0.166
$$

$$
\begin{array}{cc}
\frac{d}{d t} y(t)=-0.021 y(t)+0.025 x(t)+0.1 & x(0)=1 . \& \\
\frac{d}{d t} z(t)=-0.05 z(t)+0.02 y(t)+0.02: & y(0)=3.5 \\
z(0)=0.5
\end{array}
$$




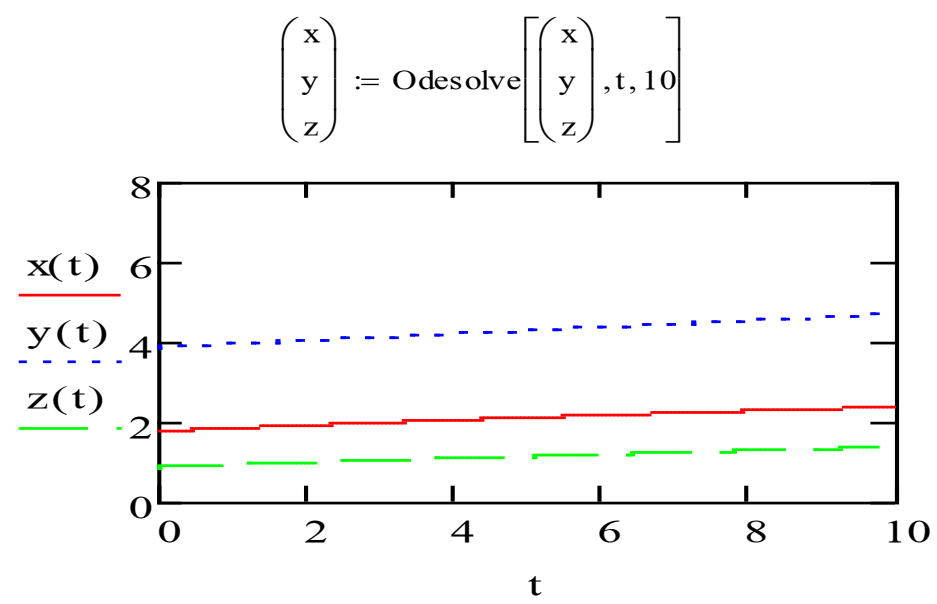

Fig. 3. System graph with initial conditions $x(0)=1,8, y(0)=3,9, z(0)=0,9$

$$
x(10)=2,402 ; y(10)=4,725 ; z(10)=1,403
$$

Thus, within ten conditional years, the university is returning to the previous indicators in terms of the composition of the teaching staff.

Let us consider a special case when in system (4-9) the free members are periodic functions, which ultimately describe some chaos in maintaining the stability of personnel, and the initial and final values of $t$ remain unchanged (Fig. 4).

Figure 4.

Given

$$
\begin{array}{cr}
\frac{d}{d t} x(t)=-0.01 \cdot x(t)+0.7 \cdot \sin (2 \cdot t) & x(0)=3 \\
\frac{d}{d t} y(t)=-0.01 \cdot y(t)+0.02 \cdot x(t)+0.09 \sin (3 \cdot t) & y(0)=6 \\
\frac{d}{d t} z(t)=-0.01 \cdot z(t)+0.001 y(t)+0.07 \cdot \cos (2 \cdot t) & z(0)=1
\end{array}
$$

$$
\left(\begin{array}{l}
x \\
y \\
z
\end{array}\right):=\operatorname{Odes} \text { olve }\left[\left(\begin{array}{l}
x \\
y \\
z
\end{array}\right), t, 10\right]
$$

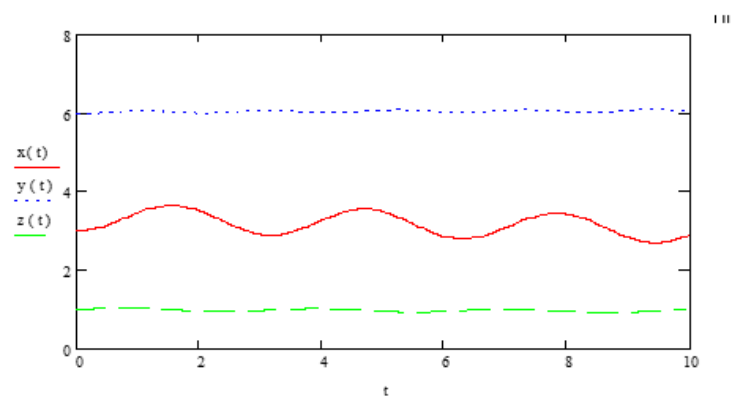

Fig. 4. Graph of the solution of the system for the functions $f(t)$ 
As a result, after a certain period, the target values of the functions will take the following numeric values: $x(10)=2,89 ; y(10)=6,054 ; z(10)=0,994$

The graph in Fig. 4 shows that if the given functions $f(x)$, где $i=1,2,3$ are periodic, then the dynamics of the number of faculty members of the university changes throughout the entire period under consideration. The graphs in Figures 1 and 2 can be used to determine the number of PPS at any time.

The state of the teaching staff-the most important component of the functioning of the university-should be constantly subjected to systematic monitoring, which identifies problems and tasks. Management involves choice, so the long-term planning stage should assess the consequences of the decisions made. At the same time, when making strategic decisions, analysis based on modern information technologies should lead to the achievement of target parameters.

For an objective, scientific forecast, it is necessary to find patterns of dynamic changes in each group of the composition of the PPP for previous years. The proposed mathematical model will make it possible to predict the change in the PPP by groups when establishing the initial conditions for the system (4) by statistical methods, as well as when checking the constant coefficients $\alpha_{i}, \beta_{i}$ and functions $f_{i}(t)$, where $i=1,2,3$. Using the MathCAD 15 software package, you can choose several options in connection with the change in the set parameters of the system. Thus, changing the control matrices A and $F$ by solving a system of ordinary differential equations has a direct impact on the solution of the system that models the process of managing the long-term development and achievement of target results of the academic potential of a branch university.

\section{Statistical analysis of the dynamics of the teaching staff by age}

Using the methods of processing statistical data, let us consider the dynamics of the distribution of frames at different age intervals. [7].

Let the result of the analysis of the staffing table be the number of teachers $n=1000$. The age group up to 30 years is formed at the expense of graduate students, graduates of their own and other universities. The second group-up to 40 years and, thus, up to 99 years. The dynamics of the average age of the university's teaching staff over the past five years (from 40 years in 2015 to 48 years in 2020) allows us to note a certain tendency to increase the average age, to the aging of the staff at the university. We will divide all teachers by age into eight categories (Table 1).

Table 1. Interval statistical series.

\begin{tabular}{|l|c|c|c|c|c|c|c|c|}
\hline & \multicolumn{9}{|c|}{ Age of the teaching staff } \\
\hline Discharges & $20-30$ & $30-40$ & $40-50$ & $50-60$ & $60-70$ & $70-80$ & $80-90$ & $90-99$ \\
\hline $\begin{array}{l}\text { Number of teaching } \\
\text { staff } m_{i}\end{array}$ & 110 & 130 & 180 & 240 & 170 & 90 & 70 & 10 \\
\hline Frequency $p_{\mathrm{i}}$ & 0,11 & 0,13 & 0,18 & 0,24 & 0,17 & 0,09 & 0,07 & 0,01 \\
\hline$b_{i}=p_{i} / h$ & 0,014 & 0,016 & 0,022 & 0,03 & 0,021 & 0,011 & 0,009 & 0,001 \\
\hline
\end{tabular}

To calculate the static distribution, taking as the "representative" of each digit its value, the interval series is replaced by a discrete static series (Table 2).

Table 2. Discrete statistical series

\begin{tabular}{|l|c|c|c|c|c|c|c|c|}
\hline Midpoint of the interval $x_{i}$ & 25 & 35 & 45 & 55 & 65 & 75 & 85 & 95 \\
\hline Observations, number $m_{i}$ & 110 & 130 & 180 & 240 & 170 & 90 & 70 & 10 \\
\hline
\end{tabular}

According to the table, we will calculate only some sample values:

In our case, the calculations give the following results: 
1. The average value of the sample.

$\bar{x}=\alpha_{1}=53,3-$ statistical analogue of mathematical expectation.

2. Sample variance.

$S=13,3$ - sample standard deviation.

Normal distribution density plot with parameters $(m, \sigma)$

$$
f(x)=\frac{1}{\sigma \sqrt{2 \pi}} e^{-\frac{(x-m)^{2}}{2 \sigma^{2}}}
$$

In our example, the form of the graph of the function (Fig. 5) gives grounds to accept the hypothesis of the normal nature of the theoretical distribution, which means that $m=\bar{x}=53,3 \quad \sigma=S=13,3$. 5):

So, as a theoretical (smoothing) distribution curve, we take the graph of the function (Fig.

$$
\text { dnorm }(\mathrm{x}, 53,12)
$$

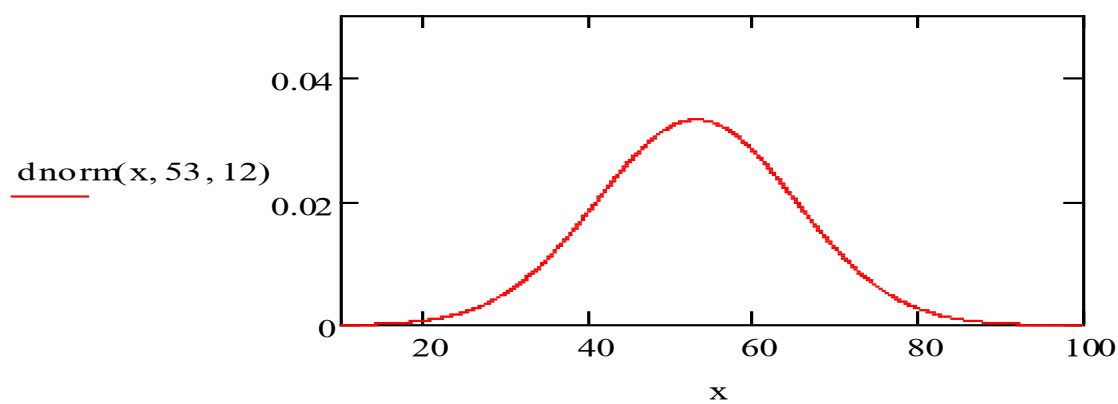

Fig. 5. The graph of the normal distribution function of the teaching staff of the university.

To check the correspondence of the chosen theoretical distribution to the experimental data, we will use the Pearson $\left(\chi^{2}\right)$, on the basis of which the empirical distribution does not contradict the hypothesis of a normal theoretical distribution with parameters $m=53,3$ и $\sigma=$ 13,3 .

Let us consider an example of the distribution of doctors of sciences, professors by age with parameters $m=61$ and $\sigma=14,3$ (Fig. 6).

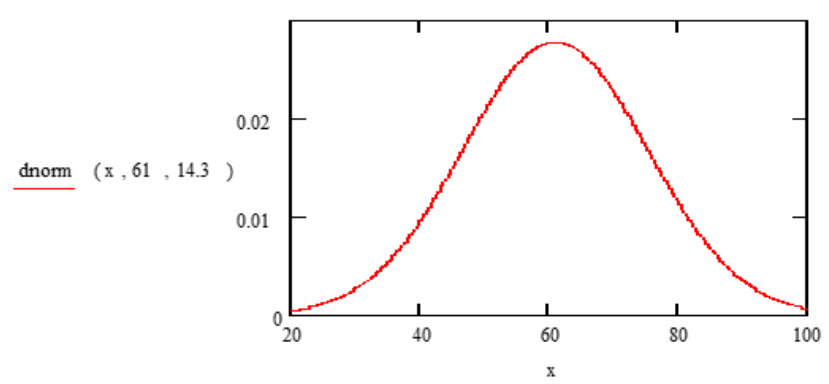

Fig. 6. Distribution of teaching staff by age.

It should be noted that in the eight railway universities studied, the distribution of teachers by age follows a normal law. However, in a number of universities there is a negative value 
of the asymmetry of distribution and an increase in the mathematical expectation or the average age of the teaching staff. From the graphs in Fig. 5,6 it follows that in technical universities there is a risk zone in the composition of the teaching staff associated with the age of teachers. The age of teachers from 70 years and older is $17 \%$ or more in railway universities in accordance with the statistical analysis (Table 1).

\section{Conclusions}

1. The constructed mathematical model reflects the real processes of sustainable development of the number of teaching staff, and the results of statistical analysis allow us to determine the values of the coefficients and functions included in the equations of the system (4) and (10).

2. Based on the analysis and changes in the structure of the teaching staff in previous years, the values of the coefficients and functions $f_{i}(t)$, where $i=1,2,3$, included in the equations of the system (4), as well as the initial conditions for predicting the sustainable development of teachers for subsequent years are determined.

3. The matrices $\mathrm{A}$ and $F$ of the coefficients of the corresponding system of linear differential equations and their free terms define the control matrices for solving the system of ordinary differential equations (4) or (10), which directly affect the solution of the system of ordinary differential equations. The analysis of management matrices and the corresponding conclusions directly affect the process of managing the long-term development and achievement of target results of the personnel potential of the branch university.

4. The constructed model allows us to determine the total number of faculty members for any period of time:

$$
N(t)=\sum_{i=1}^{n} x_{i}(t)
$$

5. The differential mathematical model can be used for practical purposes and to predict the sustainable development of the teaching staff of the university at a given time.

\section{References}

1. R. A. Kochkarov, Bulletin of the Financial Academy. Strategic Planning and Forecasting, 97 (2006)

2. G. G. Malinetsky, I. V. Kuznetsov, A.V. Podlazov, Bulletin of the Russian Academy of Sciences. On the National Scientific Monitoring System, 1 (2005)

3. G. S. Pospelov, V. A. Irikov, Program-target planning and management (1976)

4. V. E. Kozlov, E. Yu. Levina, S. V. Khusainova, L. A. Shabanova, Bulletin of ChSPU. Model of scientific and methodological support for professional growth of teachers in training, 107 (2019)

5. A. A. Samarsky, A. P. Mikhailov, Mathematical modeling. Ideas. Methods. Examples (2001)

6. V. V. Stepanov, Course of differential Equations (1950)

7. E. G. Makarov MathCAD: Training course (2009)

8. N. V. Kalganova, Bulletin of the USGUPS. Mathematical models of optimal management of material support for educational and scientific activities of industrial universities, 80 (2020) 